\title{
CHARACTERISTIC NUMBERS FOR UNORIENTED SINGULAR $G$-BORDISM
}

\author{
S. S. KHARE AND B. L. SHARMA
}

\begin{abstract}
We develop the notion of characteristic numbers for unoriented singular $G$-manifolds in a $G$-space, $G$ being a finite group, and prove their invariance with respect to unoriented singular $G$-bordism.
\end{abstract}

Thom [5] gave the notion of Stiefel Whitney numbers and Pontrjagin numbers of a manifold $M^{n}$ and proved its invariance with respect to bordism. Chung N. Lee and Arthur Wasserman [4] developed these notions for $G$ manifolds. In this note we have developed these notions for unoriented singular principal $G$-manifolds in a $G$-space, $G$ being a finite group, and proved their invariance with regard to unoriented singular $G$-bordism.

1. Characteristic numbers. Let $X$ be a finite $\mathrm{CW}$-complex with free action of $G, G$ being a finite group, and $X / G$ be again a finite $C W$-complex. Let $h^{*}$ be an equivariant cohomology theory and $h_{*}$ be the associated equivariant homology theory [1]. Let $h^{*}=H^{*} \circ A$ and $h_{*}=H_{*} \circ A$, where $A$ is a functor from the category of $G$-spaces and equivariant maps to the category of topological spaces and continuous maps, $H^{*}$ is the singular cohomology theory and $H_{*}$ is the associated singular homology theory. Let

$$
\langle,\rangle: h^{*}(X ; G) \otimes_{H^{*}(\mathrm{pt} .)} h_{*}(X ; G) \rightarrow H_{*}(\mathrm{pt} .)
$$

be the Kronecker pairing.

Let us assign to each compact $G$-manifold $W$, a class

$$
[W, \partial W] \in h_{*}(W, \partial W ; G)
$$

such that

(a) $\left[W_{1} \cup W_{2}, \partial W_{1} \cup \partial W_{2}\right]=\left[W_{1}, \partial W_{1}\right]+\left[W_{2}, \partial W_{2}\right]$,

(b) $\partial_{*}[W, \partial W]=[\partial W]$.

Suppose $\left[M^{n}, f ; G\right]$ is an element of unoriented bordism group $\mathfrak{R}_{n}(\grave{X} ; G)[3]$ and $x \in h^{*}\left(B(O, G)_{n} ; G\right), B(O, G)_{n}$ being the classifying space for $G$-vector bundles of dimension $n$. Then the $x$-characteristic number of the map $f: M^{n} \rightarrow X$ associated with an element $a^{m} \in h^{m}(X ; G)$ is defined to be

Received by the editors August 22, 1974.

AMS (MOS) subject classifications (1970). Primary 57D85.

Key words and phrases. $G$-bordism, equivariant homology, equivariant cohomology.

Copyright O 1977, American Mathematical Society 
$\left\langle\tau_{M^{n}}^{*}(x) f^{*}\left(a^{m}\right),[M]\right\rangle$, where $\tau_{M^{n}}: M^{n} \rightarrow B(O, G)_{n}$ is the tangent map.

In particular, let the equivariant cohomology $h^{*}$ be given by $h^{*}(X ; G)$ $=H^{*}\left(\left(E_{G} \times X\right) / G ; \mathbf{Z}_{2}\right)$ and $h_{*}$ be the associated equivariant homology, i.e. $h_{*}(X ; G)=H_{*}\left(\left(E_{G} \times X\right) / G ; \mathbf{Z}_{2}\right)$, where the action of $G$ on $E_{G} \times X$ is given by $g(e, x)=(g e, g x), E_{G}$ being the total space of the universal $G$-bundle. Consider the map $q: X / G \rightarrow\left(E_{G} \times X\right) / G$ given by $q([x])=[\bar{\alpha}(x), x]$, where $\bar{\alpha}$ is the map given by the following commutative diagram:

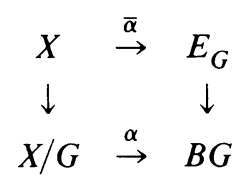

The map $q$ is homotopy equivalence. Thus

$$
h^{*}(X ; G) \stackrel{q^{*}}{\approx} H^{*}\left(X / G ; \mathbf{Z}_{2}\right) \text { and } h_{*}(X ; G) \stackrel{q_{*}^{-1}}{\approx} H_{*}\left(X / G ; \mathbf{Z}_{2}\right) .
$$

Therefore $h_{*}\left(M^{n} ; G\right) \approx H_{*}\left(M^{n} / G ; \mathbf{Z}_{2}\right)$ has a topological class, say $\sigma_{n}$, in dimension $n$.

2. Invariance of characteristic numbers. Throughout the section we will be considering equivariant cohomology $h^{*}$ to be

$$
h^{*}(X ; G)=H^{*}\left(\left(E_{G} \times X\right) / G ; \mathbf{Z}_{2}\right)
$$

and equivariant homology $h_{*}$ to be

$$
h_{*}(X ; G)=H_{*}\left(\left(E_{G} \times X\right) / G ; \mathbf{Z}_{2}\right) .
$$

THEOREM 2.1. If $\left[M^{n}, f ; G\right] \in \mathfrak{R}_{n}(X ; G)$ is zero then all the $x$-characteristic numbers of the map $f: M^{n} \rightarrow X$ associated with every $a^{m} \in h^{m}(X ; G)$ are zero.

Proof. Since $\left[M^{n}, f ; G\right] \in \mathfrak{N}_{n}(X ; G)$ is zero, $\exists$ an $(n+1)$-dimensional compact principal $G$-manifold $W^{n+1}$ and an equivariant map $F: W^{n+1} \rightarrow X$ with $\partial W^{n+1}=M^{n}$ and $F / M^{n}=f$. Let $\omega_{n+1} \in h_{n+1}\left(W^{n+1}, \partial W^{n+1} ; G\right)$ be the topological class of $W^{n+1}$. Then $\partial_{*}\left(\omega_{n+1}\right)=\sigma_{n}$. We have the following commutative diagram:

$$
\begin{array}{ccc}
h^{*}\left(B(O, G)_{n} ; G\right) & \stackrel{\tau_{M^{n}}^{*}}{\longrightarrow} & h^{*}\left(M^{N} ; G\right) \\
\uparrow j^{*} & & \uparrow i^{*} \\
h^{*}\left(B(O, G)_{n+1} ; G\right) & \stackrel{\tau_{W^{n+1}}^{*}}{\longrightarrow} & h^{*}\left(W^{n+1} ; G\right),
\end{array}
$$

where $j: B(O, G)_{n} \rightarrow B(O, G)_{n+1}$ is the map classifying $\mu_{n} \oplus 1, \mu_{n} \rightarrow B(O, G)_{n}$ being the universal $G$-vector bundle. Also we have

$$
\begin{aligned}
h^{*}\left(B(O, G)_{n} ; G\right) & =H^{*}\left(\left(E_{G} \times B(O, G)_{n}\right) / G ; \mathbf{Z}_{2}\right) \\
& =H^{*}\left(B G \times B O_{n} ; \mathbf{Z}_{2}\right) \quad[6] \\
& =H^{*}\left(B G ; \mathbf{Z}_{2}\right) \otimes H^{*}\left(B O_{n} ; \mathbf{Z}_{2}\right)
\end{aligned}
$$


and

$$
h_{*}\left(B(O, G)_{n} ; G\right)=H_{*}\left(B G ; \mathbf{Z}_{2}\right) \otimes H_{*}\left(B O_{n} ; \mathbf{Z}_{2}\right) .
$$

Thus the map $j^{*}$ is a surjection. Therefore for every $x \in h^{*}\left(B(O, G)_{n} ; G\right), \exists y$ $\in h^{*}\left(B(O, G)_{n+1} ; G\right)$ such that $j^{*}(y)=x$. Therefore

$$
\begin{aligned}
\left\langle\tau_{M^{n}}^{*}(x) f^{*}\left(a^{m}\right), \sigma_{n}\right\rangle & =\left\langle\tau_{M^{n}}^{*} j^{*}(y) f^{*}\left(a^{m}\right), \sigma_{n}\right\rangle=\left\langle i{ }^{*} \tau_{W^{n+1}}^{*}(y) i^{*} F^{*}\left(a^{m}\right), \sigma_{n}\right\rangle \\
& =\left\langle\tau_{W^{n+1}}^{*}(y) F^{*}\left(a^{m}\right), i_{*} \partial_{*}\left(\omega_{n+1}\right)\right\rangle=0 .
\end{aligned}
$$

This completes the proof of the theorem.

Consider now the map $\mu: \mathfrak{R}_{*}(X ; G) \rightarrow h_{*}(X ; G)$ defined as $\mu\left(\left[M^{n}, f ; G\right]\right)$ $=q_{*} \bar{f}_{*}\left(\bar{\sigma}_{n}\right)$, where $\bar{f}$ is the map given by the following commutative diagram:

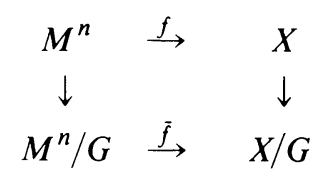

$\overline{\sigma_{n}} \in H_{n}\left(M^{n} / G ; \mathbf{Z}_{2}\right)$ being the fundamental class and let $\bar{\mu}: \Re_{*}(X / G)$ $\rightarrow H_{*}\left(X / G ; \mathbf{Z}_{2}\right)$ be the map defined by $\bar{\mu}\left(\left[N^{n}, g\right]\right)=g_{*}\left(\bar{\sigma}_{n}^{\prime}\right)$, where $\overline{\boldsymbol{\sigma}}_{n}^{\prime}$ $\in H_{n}\left(N^{n} ; \mathbf{Z}_{2}\right)$ is the fundamental class. Suppose $\phi_{*}: \mathfrak{R}_{*}(X ; G) \rightarrow \mathfrak{R}_{*}(X / G)$ is the isomorphism [3] defined as $\phi_{*}\left(\left[M^{n}, f ; G\right]\right)=\left[M^{n} / G, \bar{f}\right]$. Then $\mu$ $=q_{*} \bar{\mu} \phi_{*}$ and, therefore, $\mu$ is an epimorphism, since $\bar{\mu}$ is so [2]. For every $a \in h_{*}(X ; G)$, we select $\left[M^{n}, f ; G\right] \in \mathfrak{R}_{*}(X ; G)$ such that $\mu\left(\left[M^{n}, f ; G\right]\right)=a$. We define the $\mathfrak{N}$-module structure on $h_{*}(X ; G)$ by

$$
\left[V^{m}\right] a=\mu\left[M^{n} \times V^{m}, f^{\prime} ; G\right],
$$

for every $\left[V^{m}\right] \in \mathfrak{R}$, where the action of $G$ on $M^{n} \times V^{m}$ is defined as $g(x, y)=(g x, y)$ and $f^{\prime}: M^{n} \times V^{m} \rightarrow X$ is defined as $f^{\prime}(x, y)=f(x)$. Thus $h_{*}(X ; G) \otimes \mathfrak{N}$ is a $\mathfrak{N}$-module. Let $\left\{C_{n, i}\right\}$ be the additive base of $h_{*}(X ; G)$. Let $\left[M_{i}^{n}, f_{i} ; G\right] \in \mathfrak{N}_{*}(X ; G)$ with $\mu\left(\left[M_{i}^{n}, f_{i} ; G\right]\right)=C_{n, i}$. We define $h: h_{*}(X ; G)$ $\otimes \mathfrak{N} \rightarrow \mathfrak{N}_{*}(X ; G)$ by $h\left(C_{n, i} \otimes 1\right)=\left[M_{i}^{n}, f_{i} ; G\right]$

THEOREM 2.2. The map $h: h_{*}(X ; G) \otimes \mathfrak{R} \rightarrow \mathfrak{N}_{*}(X ; G)$, defined as above is an isomorphism.

Proof. We have the following commutative diagram:

$$
\begin{array}{ccc}
h_{*}(X ; G) \otimes \Re & \stackrel{h}{\rightarrow} & \mathfrak{N}_{*}(X ; G) \\
\downarrow q_{*}^{-1} \otimes 1_{\mathfrak{R}} & & \downarrow \phi_{*} \\
H_{*}\left(X / G ; \mathbf{Z}_{2}\right) \otimes \mathfrak{N} & \stackrel{\bar{h}}{\rightarrow} & \mathfrak{N}_{*}(X / G)
\end{array}
$$

where $\bar{h}: H_{*}\left(X / G ; \mathbf{Z}_{2}\right) \otimes \Re \rightarrow \Re_{*}(X / G)$ is defined as $\bar{h}\left(\bar{C}_{n, i} \otimes 1\right)=\left[M_{i}^{n} / G\right.$; $\bar{f}_{i}$ ], where $\bar{C}_{n, i}=q_{*}^{-1}\left(C_{n, i}\right)$. We already know that $\bar{h}$ is an isomorphism [2] and, therefore, so is $h$.

The above theorem gives the converse of Theorem 2.1 given as below. 
THEOREM 2.3. If all the characteristic numbers of an element $\left[M^{n}, f ; G\right]$ $\in \mathfrak{N}_{*}(X ; G)$ are zero, then $\left[M^{n}, f ; G\right]=0$.

Proof. Let $\mu\left(\left[M^{n}, f ; G\right]\right)=C_{n} \in h_{*}(X ; G) \quad$ and $\quad q_{*}^{-1}\left(C_{n}\right)=\bar{C}_{n}$ $\in H_{*}\left(X / G ; \mathbf{Z}_{2}\right)$. Therefore $\bar{f}_{*}\left(\overline{\boldsymbol{\sigma}}_{n}\right)=\bar{C}_{n}$. Suppose $\left\{C_{n, i}\right\}_{i \in I}$ is an additive base of $h_{n}(X ; G)$ and $C^{n, j} \in h^{n}(X ; G)$ is the cohomology class dual to $C_{n, i}$ in the sense $\left\langle\bar{C}^{n, j}, \bar{C}_{n, i}\right\rangle=\delta_{i j}$, where $q_{*}^{-1}\left(C_{n, i}\right)=\bar{C}_{n, i}$ and $q^{*}\left(C^{n, j}\right)=\bar{C}^{n, j}$. Let $C_{n}=\sum_{i \in S} \pm C_{n, i}, S$ being a finite subset of $I$. Then if $C^{n}=\sum_{i \in S} \pm C^{n, i}$, by hypothesis the $x$-characteristic number of $\left[M^{n}, f ; G\right]$ associated with $C^{n}$ $\in h^{n}(X ; G)$ is zero, that means taking $x$ to be unit class of $h^{*}\left(B(O, G)_{n} ; G\right)$,

$$
\left\langle f^{*}\left(C^{n}\right),[M]\right\rangle=0, \text { or }\left\langle f^{*}\left(C^{n}\right), q_{*}\left[\bar{\sigma}_{n}\right]\right\rangle=0,
$$

or

$$
\left\langle\left(q^{*}\right)^{-1}\left(\bar{C}^{n}\right), f_{*} q_{*}\left[\bar{\sigma}_{n}\right]\right\rangle=0, \text { or }\left\langle\left(q^{*}\right)^{-1}\left(\bar{C}^{n}\right), q_{*} \bar{f}_{*}\left[\bar{\sigma}_{n}\right]\right\rangle=0,
$$

by the following commutative diagram

$$
\begin{array}{ccc}
h_{n}\left(M^{n} ; G\right) & \stackrel{f_{*}}{\rightarrow} & h_{n}(X ; G) \\
\downarrow q_{*}^{-1} & & \downarrow q_{*}^{-1} \\
H_{n}\left(M^{n} / G ; \mathbf{Z}_{2}\right) & \stackrel{\bar{f}_{*}}{\rightarrow} & H_{n}\left(X / G ; \mathbf{Z}_{2}\right)
\end{array}
$$

Therefore $\left\langle\left(q^{*}\right)^{-1}\left(\bar{C}^{n}\right), q_{*}\left(\bar{C}_{n}\right)\right\rangle=0$, which implies that $\left\langle\bar{C}^{n}, \bar{C}_{n}\right\rangle=0$, showing that $\bar{C}_{n}=0$. Also it is easy to see that $h\left(C_{n} \otimes 1\right)=\left[M^{n}, f ; G\right]$. Since $h$ is an isomorphism and $C_{n}=0,\left[M^{n}, f ; G\right]=0$, which completes the proof of the theorem.

Theorems 2.1 and 2.3 give the invariance of characteristic numbers with regard to unoriented singular principal $G$-bordism.

\section{REFERENCES}

1. G. E. Bredon, Equivariant cohomology theories, Lecture Notes in Math., vol. 34, SpringerVerlag, Berlin and New York, 1967. MR 35 \#4914.

2. P. E. Conner and E. E. Floyd, Differentiable periodic maps, Academic Press, New York; Springer-Verlag, Berlin, 1964. MR 31 \#750.

3. S. S. Khare and B. L. Sharma, Equivariant bordism, cobordism and duality theorem (communicated).

4. C. N. Lee and A. G. Wasserman, Equivalent characteristic numbers (Proc. Second Conf. Compact Transformation Groups, 1971), Part I, Lecture Notes in Math., vol. 298, SpringerVerlag, Berlin, 1972, pp. 191-216. MR 51 \#1853.

5. R. Thom, Quelques propriétés globales des variétés différentiables, Comment Math. Helv. 28 (1954), 17-86. MR 15, 890.

6. T. tom Dieck, Faserbündel mit Gruppenoperation, Arch. Math. (Basel) 20 (1969), 136-143. MR 39 \# 6340 .

Department of Mathematics, North-Eastern Hill University, Rita Villa, Lower Lachaumiere, Shillong-793 001, Meghalaya, India 